\title{
Effects of familial hypercholesterolemia- associated genes on the phenotype of premature myocardial infarction
}

Chongyou Lee ${ }^{1,2,3+}$, Yuxia Cui ${ }^{1,2,3 \dagger}$, Junxian Song ${ }^{1,2,3}$, Sufang Li $\mathrm{L}^{1,2,3}$, Feng Zhang ${ }^{1,2,3}$, Manyan Wu ${ }^{1,2,3}$, Long Li ${ }^{1,2,3}$, Dan $\mathrm{Hu}^{1,2,3}$ and Hong Chen ${ }^{1,2,3^{*}}$

\begin{abstract}
Background: The incidence of premature myocardial infarction (PMI) has gradually increased in recent years. Genetics plays a central role in the development of PMI. Familial hypercholesterolemia $(\mathrm{FH})$ is one of the most common genetic disorders of cholesterol metabolism leading to PMI.
\end{abstract}

Objective: This study investigated the relationship between FH-associated genes and the phenotype of PMI to clarify the genetic spectrum of PMI diseases.

Method: This study enrolled PMI patients $(n=225)$ and detected the mutations in their FH-associated genes (LDLR, APOB, PCSK9, LDLRAP1) by Sanger sequencing. At the same time, patients free of PMI (non-FH patients, $n=56$ ) were enrolled as control, and a logistic regression analysis was used to identify risk factors associated with PMI. The diagnosis of FH was confirmed using "2018 Chinese expert consensus of FH screening and diagnosis" before the prevalence and clinical features of FH were analyzed.

Results: Pathogenic mutations in LDLR, APOB, PCSK9 and LDLRAP1 genes were found in 17 of 225 subjects (7.6\%), and all mutations were loss of function (LOF) and heterozygous. The genotype-phenotype relationship of patients carrying FH-associated mutations showed high heterogeneity. The logistic regression analysis showed that the smoking history, obesity and the family history of premature CHD were independent risk factors of PMI. In this study, a total of 19 patients (8.4\%) were diagnosed as $\mathrm{FH}$, and the proportion of smoking subjects in $\mathrm{FH}$ patients was higher than that in non-FH patients.

Conclusions: $\mathrm{FH}$-associated gene mutations were present in about $7.6 \%$ of Chinese patients with PMI. In addition to genetic factors, smoking history, lifestyle and other environmental factors may play a synergistic role in determining the phenotype of PMI.

Trial registration: Essential gene mutation of cholesterol metabolism in patients with premature myocardial infarction. ChiCTR-OCH-12002349.Registered 26 December 2014, http://www.chictr.org.cn/showproj.aspx?proj=7201.

Keywords: Premature myocardial infarction, Cholesterol metabolism, Familial hypercholesterolemia, Gene mutation

\footnotetext{
* Correspondence: chenhongbj@medmail.com.cn

${ }^{\dagger}$ Chongyou Lee and Yuxia Cui are contributed equally to this work.

'Department of Cardiology, Peking University People's Hospital, Xizhimen

South Rd. No.11, Xicheng district, Beijing 100044, China

${ }^{2}$ Beijing Key Laboratory of Early Prediction and Intervention of Acute

Myocardial Infarction, Peking University People's Hospital, Beijing, China

Full list of author information is available at the end of the article
}

(c) The Author(s). 2019 Open Access This article is distributed under the terms of the Creative Commons Attribution 4.0 International License (http://creativecommons.org/licenses/by/4.0/), which permits unrestricted use, distribution, and reproduction in any medium, provided you give appropriate credit to the original author(s) and the source, provide a link to the Creative Commons license, and indicate if changes were made. The Creative Commons Public Domain Dedication waiver (http://creativecommons.org/publicdomain/zero/1.0/) applies to the data made available in this article, unless otherwise stated. 


\section{Introduction}

As the most severe type of coronary artery diseases, myocardial infarction (MI) has posed a threat to public health because of its high morbidity and mortality. More than 600,000 people suffer from MI, which leads to 180,000 deaths yearly in China $[1,2]$. In recent years, the incidence of premature myocardial infarction (PMI) has gradually increased [3]. It has been shown that genetics plays a central role in the development of PMI, with its heritability estimated at approximately 63\% [4]. It was also reported that about $10-15 \%$ of PMI cases were caused by essential mutations in genes related to cholesterol metabolism [5]. Familial hypercholesterolemia $(\mathrm{FH})$ is one of the most common genetic disorders of cholesterol metabolism [6], and the mutations in $\mathrm{FH}$-associated genes, such as low-density lipoprotein receptor (LDLR), apolipoprotein $\mathrm{B}$ (APOB), proprotein convertase subtilisin/kexin type 9 (PCSK9) and lowdensity lipoprotein receptor adaptor protein 1 (LDLRAP1), can increase the plasma levels of low-density lipoprotein cholesterol (LDL-C) and lead to PMI.

A previous study carried out by the author of this study showed that the prevalence of $\mathrm{FH}$ diagnosed by genetic testing was $4.4 \%$ [7]. However, it was also found that not all clinical phenotypes of PMI matched gene mutations. Therefore, this article aimed to investigate the relationship between $\mathrm{FH}$-associated gene and the phenotype of PMI to clarify the genetic spectrum of PMI.

\section{Materials and methods}

\section{Study population}

All MI patients were enrolled at Peking University People's Hospital between May 1, 2015 and March 31, 2017. MI, including ST-segment elevation MI and non-ST-segment elevation MI, which was defined according to the Third Universal Definition of Myocardial Infarction [8]. PMI patients (age at the first MI onset: males of $\leq 55$ years old, or females of $\leq 60$ years old) were included as the experimental group, while gender matched patients free of PMI (non-PMI, age at the first MI onset: males of $\leq 55$ years old, or females of $\leq 60$ years old) were enrolled as the control group. PMI patients with incomplete clinical data or with no blood samples were excluded. The investigational protocol of this study was approved by the ethics review board of Peking University People's Hospital and was registered into the Chinese Clinical Trial Register (registration number: ChiCTR-OCH-12002349, registry URL: http://www.chictr.org.cn/showproj.aspx?proj=7201). All subjects provided written informed consent at the time of their enrollment. The investigational protocol was designed in accordance with CONSORT2010.

\section{Collection of clinical and laboratory data}

Data of PMI patients were retrieved from previously published studies to assess the clinical characteristics of
PMI [7]. The clinical characteristics of non-PMI patients, including age, sex, body mass index (BMI), and family history of premature coronary heart disease (CHD), were collected via their medical records. Laboratory examination results, such as those of routine blood test and biochemical test carried out during the first $24 \mathrm{~h}$ after hospital admission, were also obtained. The severity of CHD was assessed according to the Gensini score system described in a previous study [9]. A family history of premature $\mathrm{CHD}$ was defined as males of $<55$ years old or females of $<60$ years old in the first-degree relatives.

\section{Diagnostic criteria for $\mathrm{FH}$}

FH was diagnosed using "2018 Chinese expert consensus of FH screening and diagnosis" [10]. Adults who met 2 of the following criteria could be diagnosed as FH: (1) untreated LDL-C $\geq 4.7 \mathrm{mmol} / \mathrm{L}(180 \mathrm{mg} / \mathrm{dl})$; (2) skin/tendon xanthoma or corneal arcus in a person of $<45$ years old; (3) A first-degree relative with $\mathrm{FH}$ or premature arteriosclerotic cardiovascular disease (ASCVD). In addition, FH was also diagnosed by detecting the pathogenic mutations in LDLR, APOB, PCSK9 and LDLRAP1 genes.

\section{Collection of blood cell samples}

Peripheral venous blood samples were collected after patients were admitted into the hospital and were processed within $30 \mathrm{~min}$ of collection. Blood cells was isolated by centrifugation at $3000 \mathrm{rpm} / \mathrm{min}$ for $10 \mathrm{~min}$ and then transferred into new tubes, which were stored at $-80^{\circ} \mathrm{C}$ until use.

\section{DNA extraction}

Genomic DNA was extracted from blood cell samples using a DNeasy Blood Kit (Tianyihuyuan, Beijing, China) according to the manufacturer's protocol.

\section{Sequencing and analysis of mutations}

The entire exon region of LDLR, PCSK9, and LDLRAP1 genes, as well as the exon 26 of APOB gene (from $100 \mathrm{bp}$ to $200 \mathrm{bp}$ of p.Arg3500) located at the LDL receptor-binding site, were studied by Sanger sequencing using an $\mathrm{ABI}$ 3730-XL Genetic Analyzer (ABI, Foster City, CA).

The LOVD database (https://databases.lovd.nl/shared/ genes/), the NCBI-ClinVar database (https://www.ncbi. nlm.nih.gov/clinvar/), and the NCBI-Pubmed database (https://www.ncbi.nlm.nih.gov/pubmed) were used to determine whether the sequenced mutations were "pathogenic" or "potentially pathogenic" mutations that have been reported. Polyphen-2 software was used to predict whether a newly found mutation was pathogenic.

\section{Statistical analysis}

SPSS19.0 software was used for analysis. Measurement data with a normal distribution were represented by $\mathrm{X} \pm$ 
$\mathrm{S}$ and examined using independent samples t-test. Count data were expressed by (RQ) and examined using $\mathrm{X}^{2}$ tests. A logistic regression analysis model was used to evaluate the correlation between various risk factors and PMI. $P<0.05$ indicated significant difference.

\section{Results}

\section{Genetic phenotypes of PMI patients}

A total of 225 PMI patients meeting the inclusion criteria were collected, including 188 males (83.6\%) and 37 females (16.4\%). 19 non-synonymous variants were identified in these PMI patients, including 12 pathogenic mutations and 7 benign variants (Table 1). Among these variants, 5 pathogenic variants (LDLR c.129G $>$ C, c.1867A > T; PCSK9 c.1792G > A; LDLRAP1 c.65G > C, c. $274 \mathrm{G}>\mathrm{A}$ ) and 4 benign variants (LDLR c.928A $>\mathrm{T}$, c.2320G > A; PCSK9 c.517C > T, c.1954A > G) were discovered for the first time.
Pathogenic mutations in LDLR, APOB, PCSK9 and LDLRAP1 genes were found in 17 of the 225 subjects (7.6\%), and all pathogenic mutations were loss of function (LOF) and heterozygous. However, these mutations also included 7 PCSK9 LOF mutations. In contrast to LOF mutations in LDLR, APOB and LDLRAP1, PCSK9 LOF mutations could increase hepatic LDLR expressions and reduce circulating levels of $\mathrm{LDL}$. One patient carried LDLR(c.129G > C), PCSK9(c.277C > T) and LDLRAP1(c.2 $74 \mathrm{G}>$ A) mutations, and all other patients carried a single gene mutation. As shown in a previous study [7], the prevalence of FH was $4.4 \%$ in PMI patients diagnosed by genetic testing.

\section{Clinical phenotypes of PMI patients}

Among the 225 PMI patients, their age at MI onset was $(46.64 \pm 7.21)$ years old. In addition, the average age at MI onset in 56 non-PMI patients was $(73.73 \pm 6.97)$ years old.

Table 1 Genetic phenotypes of PMI patients

\begin{tabular}{|c|c|c|c|c|c|}
\hline Gene & Function & cDNA position & Protein position & Significance & \\
\hline LDLR & Missense & C. $129 \mathrm{G}>\mathrm{C}$ & p.Lys43Asn & likely pathogenic & Putative \\
\hline LDLR & Missense & c. $241 C>T$ & p.Arg81Cys & pathogenic & Known \\
\hline LDLR & Missense & c. $292 \mathrm{G}>\mathrm{A}$ & p.Gly98Ser & pathogenic & Known \\
\hline LDLR & Missense & c. $1525 A>G$ & p.Lys509Glu & pathogenic & Known \\
\hline LDLR & Missense & c. $1691 A>G$ & p.Asn564Ser & pathogenic & Known \\
\hline LDLR & Missense & c. $1691 A>G$ & p.Asn564Ser & pathogenic & Known \\
\hline LDLR & Missense & c. $1867 \mathrm{~A}>\mathrm{T}$ & p.lle623Phe & likely pathogenic & Putative \\
\hline LDLR & Missense & c. $2054 C>T$ & p.Pro685Leu & pathogenic & Known \\
\hline LDLR & Missense & c. $2054 C>$ T & p.Pro685Leu & pathogenic & Known \\
\hline LDLR & & $c .928 \mathrm{~A}>\mathrm{T}$ & p.lle310Phe & benign & Putative \\
\hline LDLR & & c. $1057 \mathrm{G}>\mathrm{A}$ & p.Glu353Lys & benign & Known \\
\hline LDLR & & c. $1171 \mathrm{G}>\mathrm{A}$ & p.Ala391Thr & benign & Known \\
\hline LDLR & & c. $1516 \mathrm{G}>\mathrm{A}$ & p.Val306Met & benign & Known \\
\hline LDLR & & $c .2320 G>A$ & p.Asp774Asn & benign & Putative \\
\hline PCSK9 & Missense & c. $277 \mathrm{C}>\mathrm{T}$ & p.Arg93Cys & pathogenic & Known \\
\hline PCSK9 & Missense & c. $277 \mathrm{C}>\mathrm{T}$ & p.Arg93Cys & pathogenic & Known \\
\hline PCSK9 & Missense & c. $277 \mathrm{C}>\mathrm{T}$ & p.Arg93Cys & pathogenic & Known \\
\hline PCSK9 & Missense & c. $277 \mathrm{C}>\mathrm{T}$ & p.Arg93Cys & pathogenic & Known \\
\hline PCSK9 & Missense & c. $277 \mathrm{C}>\mathrm{T}$ & p.Arg93Cys & pathogenic & Known \\
\hline PCSK9 & Missense & c. $277 \mathrm{C}>\mathrm{T}$ & p.Arg93Cys & pathogenic & Known \\
\hline PCSK9 & Missense & c. $1792 \mathrm{G}>\mathrm{A}$ & p.Ala598Thr & likely pathogenic & Putative \\
\hline PCSK9 & & C. $517 \mathrm{C}>\mathrm{T}$ & p.Pro173Ser & benign & Putative \\
\hline PCSK9 & & c. $1954 A>G$ & p.Asn652Asp & benign & Putative \\
\hline APOB & Missense & C.10579C > T & p. Arg3527Trp & pathogenic & Known \\
\hline LDLRAP1 & Missense & c. $65 G>C$ & p.Trp22Ser & likely pathogenic & Putative \\
\hline LDLRAP1 & Missense & c. $274 G>A$ & p.Val92Met & likely pathogenic & Putative \\
\hline
\end{tabular}


Compared to non-PMI patients, the PMI patients had a higher level of LDL-C or body mass index (BMI), and were more likely to have a smoking history and a family history of premature CAD (Table 2). The logistic regression analysis showed that the differentially expressed risk factors were independent predictive factors for patients with PMI (Table 3). Although the genetic factor (a family history of premature CHD) was associated with PMI $(\mathrm{OR}=2.840 ; 95 \% \mathrm{CI}: 1.075-7.503 ; P=0.035)$, its impact on PMI was relatively weak, because PMI might also be affected by BMI, smoking and other factors.

In this study, PMI patients were divided into 4 groups according to their age (Table 4). The proportions of males and patients with a smoking history were both > $50 \%$ in all age groups. However, with the increase in age, both the proportion of males and the proportion of patients with a smoking history decreased. Besides, patients younger than 30 years old had the highest level of LDL-C, highest Gensini scores and the highest incidence with a family history of PCHD.

\section{Clinical phenotypes of patients carrying mutations}

Patients carrying mutations in different genes or different mutations of the same gene showed different levels of LDL-C and CHD severity (Table 5). The LDL-C level and Gensini scores were the highest in patients carrying LDLR mutations, followed by patients carrying APOB mutations (Fig. 1).

The LDL-C level of patients carrying PCSK9 LOF mutations ranged from $1.66 \mathrm{mmol} / \mathrm{L}$ to $3.60 \mathrm{mmol} / \mathrm{L}$, but the level of LDL-C did not match the severity of CHD. For example, the LDL-C level in Case 12 was only 1.66 $\mathrm{mmol} / \mathrm{L}$, but the Gensini score of this patient was the
Table 3 Logistic regression analysis of PMI patients

\begin{tabular}{lllll}
\hline Risk factors & $\mathrm{B}$ & $\mathrm{OR}$ & $95 \% \mathrm{Cl}$ & $\mathrm{P}$ \\
\hline $\mathrm{LDL}-\mathrm{C}(\mathrm{mmol} / \mathrm{L})$ & 0.404 & 1.498 & $1.127-1.991$ & 0.005 \\
Family history of PCHD, n (\%) & 1.044 & 2.840 & $1.075-7.503$ & 0.035 \\
BMI (kg/m $\left.{ }^{2}\right)$ & 0.172 & 1.188 & $1.079-1.307$ & $<0.001$ \\
Smoking, n (\%) & 0.732 & 2.080 & $1.128-3.835$ & 0.019 \\
\hline
\end{tabular}

PMI premature myocardial infarction, $L D L-C$ low-density lipoprotein cholesterol, $P C H D$ premature coronary heart disease, $B M I$ body mass index

highest among patients carrying PCSK9 LOF mutations (Table 5). Most of these patients had a smoking history, and some of them suffered from diabetes and hypertension.

\section{Clinical phenotypes of $\mathrm{FH}$ patients}

Among the 225 PMI patients, 11 (4.9\%) patients met at least two of the Chinese criteria for $\mathrm{FH}$ diagnosis. Of these patients, $2 / 11(1.8 \%)$ patients were also diagnosed by genetic testing. At the end, a total of 19 patients $(8.4 \%)$ were diagnosed as $\mathrm{FH}$. The proportion of smoking subjects in $\mathrm{FH}$ patients was higher than that in non-FH patients (Table 6).

\section{Discussion}

MI places a heavy psychological and the socioeconomic burden on "young" patients because it greatly affects the patients' ability to work,. The registration study of acute myocardial infarction in China (CAMI) showed that PMI patients accounted for about $27.7 \%$ of all MI patients [11]. Because genetic factors play a great role in PMI, the identification of genetic variants conferring susceptibility to PMI is important for the prevention and management of this condition.

Table 2 Clinical phenotypes of patients with PMI

\begin{tabular}{|c|c|c|c|}
\hline & Patients with PMI $(n=225)$ & Non-PMl patients $(n=56)$ & $P$ value \\
\hline Male, n(\%) & $188(83.6)$ & $45(80.4)$ & 0.569 \\
\hline $\mathrm{BMI}\left(\mathrm{kg} / \mathrm{m}^{2}\right)$ & $26.71 \pm 3.51$ & $24.58 \pm 4.12$ & 0.001 \\
\hline Age of Ml onset (years) & $46.64 \pm 7.21$ & $73.73 \pm 6.97$ & $<0.001$ \\
\hline Family history of PCHD, n (\%) & $49(21.8)$ & $5(8.9)$ & 0.029 \\
\hline eGFR $\left(\mathrm{ml} / \mathrm{min} / 1.73 \mathrm{~m}^{2}\right)$ & $96.72(82.49,104.27)$ & $78.45(67.71,89.32)$ & $<0.001$ \\
\hline $\mathrm{LDL}-\mathrm{C}(\mathrm{mmol} / \mathrm{L})$ & $3.63(2.97,4.35)$ & $3.29(2.49,3.86)$ & 0.005 \\
\hline LVEF (\%) & $60.93 \pm 10.25$ & $60.67 \pm 8.75$ & 0.861 \\
\hline Gensini score & $54(34,79)$ & $58.5(45.5,83.5)$ & 0.107 \\
\hline Multivessel lesion, n(\%) & $176(78.2)$ & $48(85.7)$ & 0.212 \\
\hline Smoking, n (\%) & $153(68.0)$ & $27(48.2)$ & 0.006 \\
\hline Hyperlipemia, n (\%) & $75(33.3)$ & $15(26.8)$ & 0.347 \\
\hline Hypertension, n (\%) & $116(51.6)$ & $35(62.5)$ & 0.142 \\
\hline Diabetes, n (\%) & $83(36.9)$ & $14(25.0)$ & 0.094 \\
\hline
\end{tabular}


Table 4 Age-related Characteristics of PMl patients

\begin{tabular}{lllll}
\hline & Age $\leq 30(n=9)$ & $30 \leq$ age $<40(n=34)$ & $40 \leq$ age $<50(n=105)$ & $50 \leq$ age $<60(n=77)$ \\
\hline Male, $\mathrm{n}(\%)$ & $9(100)$ & $34(100)$ & $93(88.6)$ & $52(67.5)$ \\
Age at Ml onset (years) & $28.33 \pm 1.58$ & $37.74 \pm 3.02$ & $46.33 \pm 2.91$ & $53.57 \pm 2.21$ \\
BMI (kg/m²) & $29.17 \pm 4.53$ & $28.42 \pm 3.90$ & $26.81 \pm 3.64$ & $25.52 \pm 2.44$ \\
Family history of PCHD, $\mathrm{n}(\%)$ & $5(55.6)$ & $11(32.4)$ & $17(16.2)$ & $16(20.8)$ \\
Smoking, $\mathrm{n}(\%)$ & $9(100)$ & $27(79.4)$ & $73(69.5)$ & $44(57.1)$ \\
LDL-C (mmol/L) & $3.98(3.87,5.42)$ & $3.67(3.18,4.36)$ & $3.61(2.79,4.34)$ & $3.65(3.10,4.40)$ \\
LVEF (\%) & $52.16 \pm 11.96$ & $58.92 \pm 10.25$ & $60.96 \pm 10.56$ & $62.81 \pm 9.04$ \\
Gensini scores & $78(38,90)$ & $55(25.25,86.76)$ & $62(56.35,68.15)$ & $53(35,78)$ \\
Multivessel lesion, $\mathrm{n}(\%)$ & $6(66.7)$ & $27(79.4)$ & $84(80)$ & $59(76.6)$
\end{tabular}

$P M I$ premature myocardial infarction, $B M I$ body mass index, $P C H D$ premature coronary heart disease, $L D L-C$ low-density lipoprotein cholesterol, $L V E F$ left ventricular ejection fraction

In this study, it was observed that $7.6 \%$ PMI patients carried $\mathrm{FH}$-associated mutations and the value was higher than that reported in the study by Khera et al., who showed that a familial hypercholesterolemia mutation was present in 36 of 2081 (1.7\%) patients with early-onset myocardial infarction [12]. The above difference may be attributed to the different geographical regions and nationalities studied in the two reports [13]. In this study, LDLR gene mutations made up the vast majority of all mutations. However, because all LDLRAP1 mutations are heterozygous, they are hence not pathogenic.

PCSK9 LOF mutations (PCSK9 c.277C > T, c.1792G > A) were also found in this study. In particular, as a mutation previously reported only in the Japanese population, the c. $277 \mathrm{C}>\mathrm{T}$ mutation can decrease the level of LDL-C and reduce the risk of ASCVD [14]. However, the patients in this study did not show lower levels of LDL-C and its reason might be their lifestyle and other environmental factors. In this study, the logistic regression analysis showed that smoking, obesity and family history of premature CHD were independent risk factors for PMI, suggesting that the lifestyle played an important role in the onset of PMI.

Men dominated PMI patients in most studies on PMI, including this study $[15,16]$, which may be attributed to the misperception that females are 'protective' against cardiovascular diseases. Previous studies have also

Table 5 Clinical phenotypes of LDLR, APOB, PCSK9 and LDLRAP1 gene mutations

\begin{tabular}{|c|c|c|c|c|c|c|}
\hline Case & Gene & Function & Exon & Sites of the Mutation & LDL-C (mmol/L) & Gensini Scores \\
\hline Case1 & LDLR & Missense & 3 & c. $241 C>T$ & 4.86 & 40 \\
\hline Case2 & LDLR & Missense & 3 & c. $292 \mathrm{G}>\mathrm{A}$ & 3.89 & 48 \\
\hline Case3 & LDLR & Missense & 10 & c. $1525 \mathrm{~A}>\mathrm{G}$ & 8.00 & 54 \\
\hline Case4 & LDLR & Missense & 11 & c. $1691 A>G$ & 4.35 & 128 \\
\hline Case5 & LDLR & Missense & 11 & c. $1691 \mathrm{~A}>\mathrm{G}$ & 7.25 & 100 \\
\hline Case6 & LDLR & Missense & 13 & c.1867A > T & 5.72 & 104 \\
\hline Case7 & LDLR & Missense & 14 & c. $2054 C>T$ & 7.74 & 78 \\
\hline Case8 & LDLR & Missense & 14 & c. $2054 C>T$ & 6.37 & 157 \\
\hline Case9 & APOB & Missense & 26 & c. $10579 C>T$ & 4.93 & 57 \\
\hline Case10 & PCSK9 & Missense & 2 & c. $277 \mathrm{C}>\mathrm{T}$ & 3.26 & 40 \\
\hline Case11 & PCSK9 & Missense & 2 & c. $277 \mathrm{C}>\mathrm{T}$ & 2.68 & 40 \\
\hline Case12 & PCSK9 & Missense & 2 & c. $277 \mathrm{C}>\mathrm{T}$ & 1.66 & 168 \\
\hline Case13 & PCSK9 & Missense & 2 & $c .277 C>T$ & 3.46 & 12 \\
\hline Case14 & PCSK9 & Missense & 2 & c. $277 \mathrm{C}>\mathrm{T}$ & 3.02 & 65 \\
\hline Case15 & PCSK9 & Missense & 3 & c. $1792 \mathrm{G}>\mathrm{A}$ & 3.60 & 117 \\
\hline Case16 & LDLRAP1 & Missense & 1 & c. $65 \mathrm{G}>\mathrm{C}$ & 2.66 & 54 \\
\hline Case17 & LDLR+PCSK9+LDLRAP1 & & & & 2.50 & 62 \\
\hline
\end{tabular}



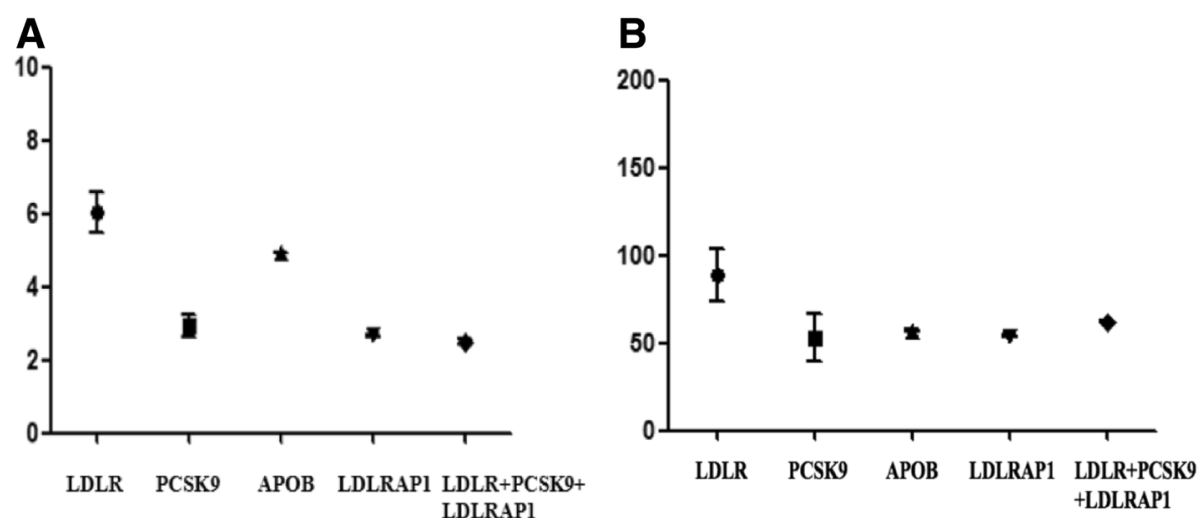

Fig. 1 LDL-C levels and Gensini scores of patients carrying mutations. LDLR, low-density lipoprotein receptor; APOB, apolipoprotein B; PCSK9, proprotein convertase subtilisin/kexin type 9; LDLRAP1, low-density lipoprotein receptor adaptor protein 1

shown that PMI patients were usually cigarette smokers and the proportion of PMI smokers increased over a decreasing age $[17,18]$, which was consistent with the result of this research. Since patients younger than 30 years were found to have more serious coronary artery lesions, both genetic (family history of PCHD) and environmental (smoking) factors play an important role in the onset of PMI.

The genotype-phenotype relationship of patients with $\mathrm{FH}$-associated mutations showed high heterogeneity. Numerous studies have demonstrated that the carriers of LDLR mutations had the highest levels of LDL-C [19, 20], which was consist with the result of this research that showed the median LDL-C level in carriers of LDLR variants was $5.72 \mathrm{mmol} / \mathrm{L}$ and was higher than that in the carriers of other mutations. In addition, the coronary lesions in carriers of LDLR variants were more severe than those in carriers of other mutations. However, patients carrying the same mutation also showed obviously different levels of LDL-C and CAD severity. Such clinical heterogeneity might be attributed to the following reasons. On the one hand, the penetrance of these genes was not 100\%; on the other hand, the different diets and lifestyles adopted by different patients might affect their clinical phenotypes.

In this study, the "2018 Chinese expert consensus of FH screening and diagnosis" was used first to diagnose FH. Only 2/11(1.8\%) patients with clinical FH carried $\mathrm{FH}$-associated gene mutations and the percentage was lower than that reported in a previous study, suggesting that different diagnostic criteria of FH may lead to different prevalence values of $\mathrm{FH}$. Most studies have also

Table 6 Clinical phenotypes of FH patients

\begin{tabular}{|c|c|c|c|}
\hline & FH patients $(n=19)$ & Non-FH patients $(n=206)$ & $P$ value \\
\hline Male, n(\%) & $17(89.5)$ & $171(83.0)$ & 0.467 \\
\hline $\mathrm{BMI}\left(\mathrm{kg} / \mathrm{m}^{2}\right)$ & $27.82 \pm 5.28$ & $26.60 \pm 3.30$ & 0.334 \\
\hline Age at Ml onset (years) & $46.68 \pm 7.97$ & $50.43 \pm 7.91$ & 0.050 \\
\hline Family history of PCHD, n (\%) & $11(57.9)$ & $38(18.4)$ & $<0.001$ \\
\hline $\mathrm{eGFR}\left(\mathrm{ml} / \mathrm{min} / 1.73 \mathrm{~m}^{2}\right)$ & $93.95(76.40,101.71)$ & $97.37(84.17,104.48)$ & 0.435 \\
\hline LDL-C (mmol/L) & $4.93(4.80,6.37)$ & $3.58(2.95,4.21)$ & $<0.001$ \\
\hline LVEF (\%) & $61.31 \pm 12.22$ & $60.90 \pm 10.08$ & 0.869 \\
\hline Gensini scores & $62(48,92)$ & $53.5(32,77.25)$ & 0.087 \\
\hline Tendon xanthoma/corneal arcus n(\%) & $0(0)$ & $0(0)$ & 1.000 \\
\hline Multivessel lesion, n(\%) & $15(78.9)$ & $161(78.2)$ & 0.936 \\
\hline Smoking, n (\%) & $17(89.5)$ & $136(66.0)$ & 0.036 \\
\hline Hyperlipemia, n (\%) & $11(57.9)$ & $64(31.1)$ & 0.018 \\
\hline Hypertension, n (\%) & $4(21.1)$ & $112(54.4)$ & 0.005 \\
\hline Diabetes, n (\%) & $7(36.8)$ & 76 (36.9) & 0.996 \\
\hline
\end{tabular}

FH familial hypercholesterolemia, $L D L-C$ low-density lipoprotein cholesterol, $B M I$ body mass index, $L V E F$ left ventricular ejection fraction. All $P$ values represent comparisons between PMI patients and non-PMI patients. Comparisons between groups were performed with student's t-test for continuous variables and with Chi-square test for categorical variables. $P<0.05$ was considered statistically significant 
found that $20-60 \%$ of subjects with phenotypic FH did not carry a causative mutation in LDLR, APOB, PCSK9 or LDLRAP1 genes, suggesting that phenotypic FH may also be induced by multiple small-effect common variants, mutations in unknown $\mathrm{FH}$-associated genes, or environmental factors [21, 22]. None of the FH patients in this study had Tendon xanthoma/corneal arcus, which was consistent with the results of previous studies [23, 24].

Nevertheless, several limitations in this study need to be addressed. Firstly, the sample size in this study is small and may cause a statistical bias. Secondly, the approach used in this study to annotate missense variants by prediction algorithms may lead to misclassification in some cases, and the predicted variants need to be validated through in vitro functional experiments. Thirdly, only $4 \mathrm{FH}$-associated common genes were detected in this study, while some other rare $\mathrm{FH}$-associated genes, including APOE and STAP1, were not detected.

In conclusion, $\mathrm{FH}$-associated gene mutations were present in about $7.6 \%$ of Chinese PMI patients. In addition to genetic factors, smoking history, lifestyle and other environmental factors may play a synergistic role in determining the phenotype of PMI.

\section{Abbreviations \\ APOB: Apolipoprotein B; ASCVD: Arteriosclerotic cardiovascular disease; BMI: Body mass index; CHD: Coronary heart disease; FH: Familial hypercholesterolemia; LDL-C: Low-density lipoprotein cholesterol; LDLR: Low- density lipoprotein receptor; LDLRAP1: Low-density lipoprotein receptor adaptor protein 1; MI: Myocardial infarction; PCSK9: Proprotein convertase subtilisin/kexin type 9; PMl: Premature myocardial infarction}

\section{Acknowledgements}

The authors would like to acknowledge all staffs in Peking University People's Hospital for their help.

\section{Funding}

This study was supported by the National Natural Science Foundation of China $(81770356,81470473$,$) , Capital Health Research and Development of$ Special Funds (No. 2016-2-4083).

\section{Availability of data and materials}

The datasets used in the current study are available from the corresponding author upon request.

\section{Authors' contributions}

$C L$ and YC wrote the manuscript; SL and JS prepared Tables 1, 2, 3, 4, 5; MW prepared Fig. 1; FZ, LLI and DH modified the manuscript; HC designed the study and modified the manuscript. All authors read and approved the final manuscript.

\section{Ethics approval and consent to participate}

Subjects have given their written informed consent. The study protocol has been approved by the research institute's committee on human research.

\section{Consent for publication}

All authors have given consent for the publication of this paper.

\section{Competing interests}

The authors declare that they have no competing interests.

\section{Publisher's Note}

Springer Nature remains neutral with regard to jurisdictional claims in published maps and institutional affiliations.

\section{Author details}

${ }^{1}$ Department of Cardiology, Peking University People's Hospital, Xizhimen South Rd. No.11, Xicheng district, Beijing 100044, China. ${ }^{2}$ Beijing Key Laboratory of Early Prediction and Intervention of Acute Myocardial Infarction, Peking University People's Hospital, Beijing, China. ${ }^{3}$ Center for Cardiovascular Translational Research, Peking University People's Hospital, Beijing, China.

Received: 30 January 2019 Accepted: 1 April 2019

Published online: 11 April 2019

\section{References}

1. Zhou Y, Yao X, Liu G, Jian W, Yip W. Level and variation on quality of care in China: a cross-sectional study for the acute myocardial infarction patients in tertiary hospitals in Beijing. BMC Health Serv Res. 2019;19(1):43.

2. Zhang Q, Zhao D, Xie W, Xie X, Guo M, Wang M, et al. Recent trends in hospitalization for acute myocardial infarction in Beijing: increasing overall burden and a transition from ST-segment elevation to non-ST-segment elevation myocardial infarction in a population-based study. Medicine (Baltimore). 2016:95(5):e2677.

3. Titov BV, Osmak GJ, Matveeva NA, Kukava NG, Shakhnovich RM, Favorov AV, et al. Genetic risk factors for myocardial infarction more clearly manifest for early age of first onset. Mol Biol Rep. 2017;44(4):315-21.

4. Fischer M, Broeckel U, Holmer S, Baessler A, Hengstenberg C, Mayer B, et al. Distinct heritable patterns of angiographic coronary artery disease in families with myocardial infarction. Circulation. 2005;111:855-62.

5. Roberts R. Genetics of premature myocardial infarction. Genetics. 2008;10: 186-93.

6. Elkins JC, Fruh S. Early diagnosis and treatment of familial hypercholesterolemia. Nurse Pract. 2019;44(2):18-24.

7. Cui Y, Li S, Zhang F, Song J, Lee C, Wu M, et al. Prevalence of familial hypercholesterolemia in patients with premature myocardial infarction. Clin Cardiol. 2019:42(3):385-90.

8. White HD, Thygesen K, Alpert JS, Jaffe AS. Clinical implications of the third universal definition of myocardial infarction. Heart. 2014;100(5):424-32.

9. Li S, Zhang Y, Xu RX, Guo YL, Zhu CG, Wu NQ, et al. Proprotein convertase subtilisin-kexin type 9 as a biomarker for the severity of coronary artery disease. Ann Med. 2015:47:386-93.

10. Gao W, Chen H, An J, Che WL, Chen JY, Dong SH, et al. Chinese expert consensus of FH screening and diagnosis. Chinese J Cardiovasc Dis. 2018; 46(2):99-103.

11. Xiaojin G, Jingang $Y$, Yuejin Y, Li W, Xu HY, Wu Y, et al. Age-related coronary risk factors in Chinese patients with acute myocardial infarction. Natl Med J China. 2016:96:3251-6.

12. Khera AV, Chaffin M, Zekavat SM, Collins RL, Roselli C, Natarajan P, et al. Whole-genome sequencing to characterize monogenic and polygenic contributions in patients hospitalized with early-onset myocardial infarction. Circulation. 2019;139(13):1593-1602.

13. Sun D, Zhou BY, Li S, Sun NL, Hua Q, Wu SL, et al. Genetic basis of index patients with familial hypercholesterolemia in Chinese population: mutation spectrum and genotype-phenotype correlation. Lipids Health Dis. 2018; 17(1):252

14. Miyake $Y$, Kimura R, Kokubo Y, Okayama A, Tomoike $H$, Yamamura $T$, et al. Genetic variants in PCSK9 in the Japanese population: rare genetic variants in PCSK9 might collectively contribute to plasma LDL cholesterol levels in the general population. Atherosclerosis. 2008;196(1):29-36.

15. Tamrakar R, Bhatt YD, Kansakar S, Bhattarai M, Shaha KB, Tuladhar E. Acute myocardial infarction in young adults: study of risk factors, angiographic features and clinical outcome. NHJ. 2013;10:12-6.

16. Abed MA, Eshah NF, Moser DK. Risk profile of myocardial infarction in young versus older adults. Heart Lung. 2018;47(3):226-30.

17. Larsen GK, Seth M, Gurm HS. The ongoing importance of smoking as a powerful risk factor for ST-segment elevation myocardial infarction in young patients. JAMA Intern Med. 2013:173:1261-2.

18. Shukla AN, Jayaram AA, Doshi D, Patel P, Shah K, Shinde A, et al. The young myocardial infarction study of the Western Indians: Youth Registry Glob Heart 2019 
19. Raal FJ, Sjouke B, Hovingh GK, Isaac BF. Phenotype diversity among patients with homozygous familial hypercholesterolemia: a cohort study. Atherosclerosis. 2016;248:238-44.

20. Cuchel M, Bruckert E, Ginsberg H, Raal FJ, Santos RD, Hegele RA, et al. Homozygous familial hypercholesterolaemia: new insights and guidance for clinicians to improve detection and clinical management. A position paper from the consensus panel on familial Hypercholesterolaemia of the European atherosclerosis society. Eur Heart J. 2014;35:2146-57.

21. Bañares V, Corral P, Medeiros A, Araujo MB, Lozada A, Bustamante J, et al. Preliminary spectrum of genetic variants in familial hypercholesterolemia in Argentina. J Clin Lipidol. 2017;11:524-31.

22. Durst R, Ibe U, Shpitzen S, Schurr D, Eliav O, Futema M, et al. Molecular genetics of familial hypercholesterolemia in Israel-revisited. Atherosclerosis. 2017;257:55-63.

23. Pimstone SN, Sun XM, du Souich C, Frohlich JJ, Hayden MR, Soutar AK Phenotypic variation in hetero- zygous familial hypercholesterolemia: a comparison of Chinese patients with the same or similar mutations in the LDL receptor gene in China or Canada. Arterioscler Thromb Vasc Biol. 1998; 18:309-15.

24. Tomlinson B, Hu M, Chow E. Current status of familial hypercholesterolemia in Chinese populations. Curr Opin Lipidol. 2019.

Ready to submit your research? Choose BMC and benefit from:

- fast, convenient online submission

- thorough peer review by experienced researchers in your field

- rapid publication on acceptance

- support for research data, including large and complex data types

- gold Open Access which fosters wider collaboration and increased citations

- maximum visibility for your research: over $100 \mathrm{M}$ website views per year

At BMC, research is always in progress.

Learn more biomedcentral.com/submissions 